\title{
Testicular Sperm Extraction Techniques in Subfertile Males
}

\author{
Robin Bahadur Basnet ${ }^{1}$, Mira Thapa', Rashmi Shrish', Preeti Bista ${ }^{2}$ \\ ${ }^{1}$ Department of Urology, National Academy of Medical Sciences, Bir Hospital, Kathmandu Nepal \\ ${ }^{2}$ Department of Infertility, Nepal International Fertility and Laparoscopy Center, Lalitpur Nepal
}

\section{ABSTRACT}

Introduction: Assisted conception is an option for subfertile couples. Surgical sperm retrieval by testicular sperm aspiration and testicular sperm extraction are widely used safe techniques to yield sperm for intracytoplasmic sperm injection. Experience with these techniques is presented.

Materials and Methods: A retrospective study of testicular sperm retrieval for assisted reproduction is presented. Testicular sperm aspiration is attempted on all azoospermic males with normal sexual characteristics. Testicular sperm extraction is attempted on consenting patients where aspiration has failed. Donor sperm injection is done to oocyte of a spouse on the failure of both techniques.

Results: Sixty-six percent of males had adequate numbers and characters of viable spermatozoa on testicular sperm aspiration. Further $53.3 \%$ of patients where aspiration failed yielded spermatozoa on testicular sperm extraction. Thus overall $77.1 \%$ of patients with azoospermia benefitted from testicular sperm retrieval techniques.

Conclusions: Testicular sperm aspiration is a technically easy, quick, safe, and cheap method of sperm retrieval. Testicular sperm extraction is a safe adjunct to aspiration with better yield.

\section{Correspondence:}

Robin Bahadur Basnet, MBBS, MS, MCh Associate Professor, Department of Urology

National Academy of Medical Sciences, Bir Hospital, Kathmandu, Nepal

ORCID ID: 0000-0001-9899-3095

Email: robinbasnet@gmail.com

Submitted: $1^{\text {st }}$ April 2020 Accepted: $18^{\text {th }}$ June 2020

Source of Support: None Conflict of Interest: None

Citation: Basnet RB, Thapa M, Shrish R, Bista P. Testicular sperm extraction techniques in subfertile males. NMJ 2020;3(1):276-8. DOI $10.3126 / \mathrm{nmj}$. v3i1.28296

Keywords: Assisted reproduction; In-vitro fertilization; Sperm aspiration; Sperm extraction

\section{INTRODUCTION}

A man is considered sterile and cannot father his genetic offspring if no sperm are detected in different locations of the testes. Under such circumstances, the use of donor sperm or adoption are the only available options remaining for the couple to have a child. ${ }^{1}$ Subfertility affects one in 20 men. $^{2}$ Abnormal semen quality or sexual dysfunction are contributing factors in about half of subfertile couples. ${ }^{2}$ A couple with subfertility of longer than three years, or with a non-reversible form of subfertility is unlikely to conceive spontaneously and should be advised for assisted conception. $^{2}$ Surgical sperm retrieval for intracytoplasmic sperm injection is indicated in obstructive azoospermia where spermatogenesis is usually normal, or non-obstructive azoospermia where spermatogenesis is present on biopsy. ${ }^{2}$ Results of retrieved testicular spermatozoa for intracytoplasmic sperm injection using surgically retrieved sperm are similar to those where ejaculated sperm is used., ${ }^{2,3}$ We present results of our experience with testicular sperm aspiration (TESA) and testicular sperm extraction (TESE) techniques.

\section{MATERIALS AND METHODS}

It is a retrospective study of 50 consecutive testicular sperm retrieval techniques done at Nepal International Fertility and Laparoscopy Center between January 2016 and August 2019. Informed consent was taken from all patients. The cohort comprised of males with either primary or secondary infertility, with documentation of azoospermia on semen analysis. Clinical examination included evaluation of gonadal development, secondary sexual characters, and hormonal evaluation. 
Azoospermia was reconfirmed a week before testicular sperm by centrifugation $(1200 \mathrm{rpm})$ of ejaculate for 10 minutes and meticulous examination of the pellet under a microscope (400x). Testicular sperm retrieval was performed concomitant with oocyte retrieval from the female partner. All couples were counseled about the availability of donor insemination if the yield of testicular sperm extraction was negative, to avoid oocyte wastage.

After oocyte retrieval from the spouse, TESA was performed on the male partner and the yield was examined immediately in the laboratory. In subjects with an adequate number and physical characteristics of spermatozoa, they were prepared for intracytoplasmic sperm injection (ICSI). In subjects with unsatisfactory sperm characteristics and those consenting, TESE was performed. Donor insemination was done to consenting partners with negative TESE. Intracytoplasmic injection of each successful sperm extracted was done to the corresponding oocyte, producing two to six embryos from each couple. A positive result with ICSI was considered when at least one viable embryo was produced on day 3 or day 5 .

Intravenous injection of 1 gram Ceftriaxone was given to all subjects one hour before the procedure. TESA was performed under local anesthesia (2\% Lignocaine). $2 \mathrm{~mL}$ lignocaine was instilled at the site of puncture and another $5 \mathrm{~mL}$ was instilled at the spermatic cord. Percutaneous puncture, using an 18 gauze butterfly needle connected to a $10 \mathrm{~mL}$ syringe, with prefilled $0.5 \mathrm{~mL}$ human tubular fluid medium was done directed at the center of the testis. On obtaining adequate depth within the testis, negative suction pressure was applied to the syringe. The needle was partially withdrawn and again inserted at various directions to obtain more tissue from the depth of the testis. Similar steps were repeated at the upper and lower poles keeping needle at the same puncture site on the scrotum and using different syringes.

TESE was also performed under local anesthesia. Another $3 \mathrm{~mL}$ lignocaine was instilled at the site of the incision. A two-centimeter long incision was made vertically on the scrotum and layers of scrotum were dissected up to tunica albuginea. $5 \mathrm{~mm}$ incisions were made on tunica albuginea at the center and upper and lower poles and testis squeezed gently to extrude testicular tissue, which was excised and transferred to the laboratory in human tubular fluid media for sperm retrieval. Tunica albuginea was closed with 5/0 Polypropylene and other layers of the scrotum were closed with 3/0 Polyglactin. All patients were discharged two hours after the procedure with Tablet Ibuprofen and Paracetamol to be taken twice daily for three days and to come for follow up after a week or immediately in case of swelling of the scrotum. Data were entered in Microsoft Excel and analysed.

\section{RESULTS}

A total of 50 consecutive patients who underwent testicular sperm extraction as part of assisted reproduction were evaluated. The cohort comprised of 38 patients with primary infertility and 12 patients with secondary infertility. Six patients with secondary infertility had a history of vasectomy and failed recanalization. One patient of secondary infertility had a history of transurethral bladder neck incision. Primary infertile males were younger (age $31.4 \pm 6.2$ years) than secondary infertile (age $41.9 \pm 4.8$ years) Ultrasonic evidence of varicocele was present in twelve primary infertile patients and four secondary infertile patients. None of the patients had a history of tuberculosis. Sixteen (42\%) of primary infertile and four $(33 \%)$ of secondary infertile patients were smokers. Eight (21\%) of primary infertile and six $(50 \%)$ of secondary infertile were diabetics and eleven (29\%) and six $(50 \%)$, respectively, were hypertensives under medication.

The outcome of testicular retrieval is outlined in Figure 1. Following TESA, 13 (66\%) of males had adequate numbers and characters of viable spermatozoa which were isolated and used for ICSI. Out of 14 patients who did not yield spermatozoa, 15 consented for TESE and underwent the procedure. Eight (53.3\%) of the TESE tissue yielded spermatozoa for ICSI. Five out of the remaining seven opted for donor insemination. Among the successful TESA patients, 31 (94\%) had positive results with ICSI, six (75\%) with TESE, and four (80\%) with donor insemination. Overall, $62 \%$ had positive results with TESA alone, which improved overall $77.1 \%$ after TESE was done on patients who had negative TESA.

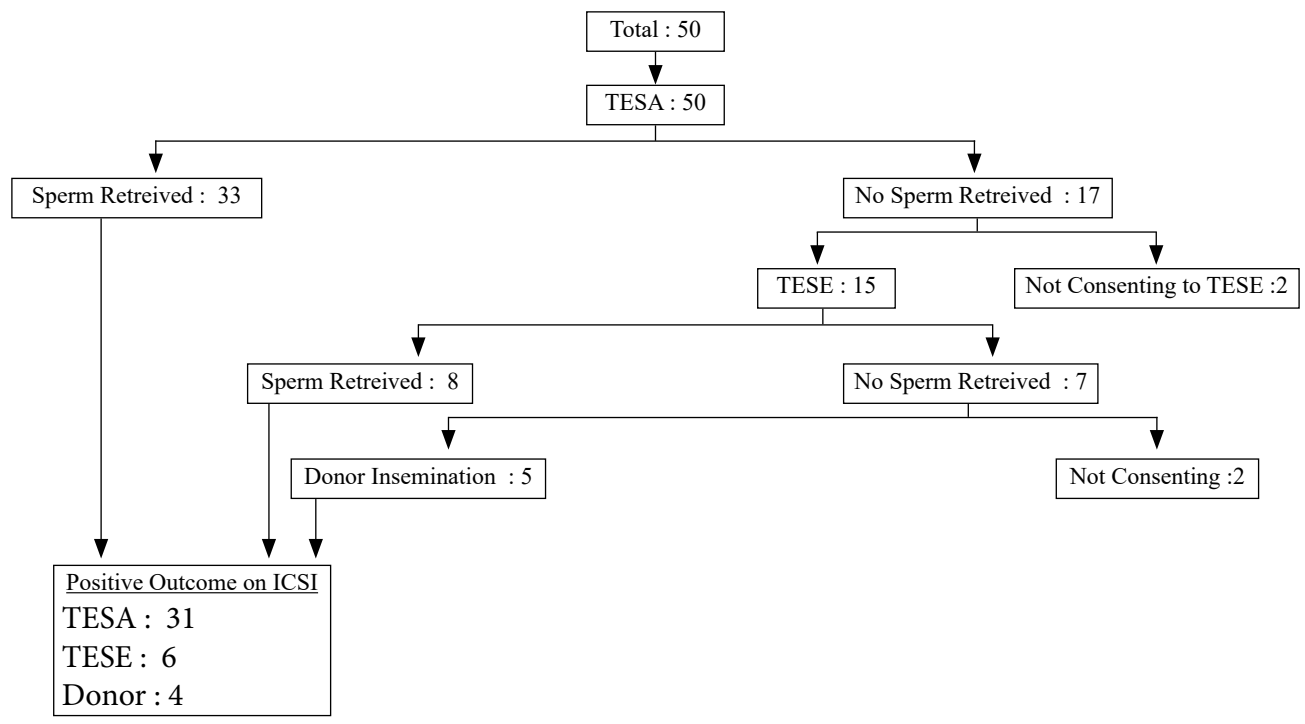

Figure 1: Outcome of primary testicular extraction procedures 
Among the cohort in this study, 41 patients (82\%) were subjected to fresh or frozen embryo transfer after intracytoplasmic sperm injection from either TESA, TESE, or donor insemination. Thirty-two patients had positive pregnancy results and thirty of them continued to full term. Two patients with negative results after TESA alone opted for donor insemination of frozen oocyte and both continued to full term. Both patients with negative TESE results did not consent for donor insemination. One patient with a failed result on donor insemination also did not consent for further treatment. Seven of nine patients with negative pregnancy results on the first IVF had second IVF with frozen embryo from index procedure and four of them continued to full term. Thus we had an overall successful outcome in thirty-six patients, nine patients discontinued treatment in between and we failed in five patients to achieve pregnancy despite all attempts at assisted reproduction.

\section{DISCUSSION}

Historically, a testicular biopsy was one of the investigations of azoospermia, but with the development of assisted reproductive techniques, it is a method of sperm retrieval for ICSI. Mature sperm cells can be found in approximately $50 \%$ of the testes of men with nonobstructive azoospermia. Even when sperm cells exist, they may not be present in all samples as testicular tissue structure is not homogenous and there may be only focal spermatogenesis. ${ }^{4}$ Since there is no reliable technique to predict sperm production and location in the testis, we aspirated testis from three different locations during TESA as well as TESE.

There have been various studies comparing TESA and TESE. Hauser et $\mathrm{al}^{1}$ compared the two procedures in the same testes in nonobstructive azoospermia. They found TESE has a better sperm recovery rate. Similar studies in both obstructive and nonobstructive azoospermia have shown mixed results, with TESE being superior in some studies ${ }^{5-8}$ while others ${ }^{9,10}$ have demonstrated similar results with both procedures. Mercan et $\mathrm{al}^{11}$ and Khadra et $\mathrm{al}^{12}$ performed TESE only after failure of TESA. They had similar findings to ours, where TESE yielded spermatozoa even when TESA failed.

TESA is technically easier, requiring fewer surgical skills and training. It is associated with no surgical scars or sutures. TESE requires a well-equipped operation theater and trained surgeon. ${ }^{3}$ Pain, intratesticular bleeding, hematoma, and infection have been reported as complications in both the procedures. ${ }^{13-15}$ However, we encountered none in our cohort. The advantages of the techniques also include cryopreservation of spermatozoa for future use. In our center, we routinely preserve excess embryo.

\section{CONCLUSIONS}

In males with azoospermia, testicular sperm aspiration is a technically easy, quick, safe, and cheap method of sperm retrieval. Testicular sperm extraction is a safe adjunct to aspiration with better yield.

\section{REFERENCES}

1. Hauser R, Yogev, L, Paz G et al. Comparison of Efficacy of Two Techniques for Testicular Sperm Retrieval in Nonobstructive Azoospermia: Multifocal Testicular Sperm Extraction Versus Multifocal Testicular Sperm Aspiration. Journal of Andrology 2006;27:28-33. Crossref

2. Hirsh A. Male subfertility. BMJ. 2003;32:669-72. Crossref

3. Salihu HM, Aliyu MH. Sperm retrieval in infertile males: comparison between testicular sperm extraction and testicular sperm aspiration techniques. Wien Klin Wochenschr. 2003;115(11):370-9. Crossref

4. Hauser R, Botchan A, Amit A, Yosef DB, Gamzu R, Paz G, et al. Multiple testicular sampling in non-obstructive azoospermia - is it necessary? Hum Reprod. 1998;13:3081-5. Website

5. Ezeh UI, Moore HD, Cooke ID. A prospective study of multiple needle biopsies versus a single open biopsy for testicular sperm extraction in men with nonobstructive azoospermia. Hum Reprod. 1998;13:3075-80. Crossref

6. Friedler S, Raziel A, Strassburger D, et al . Testicular sperm retrieval by percutaneous fine needle sperm aspiration compared with testicular sperm extraction by open biopsy in men with nonobstructive azoospermia. Hum Reprod. 1997;12:1488 - 93. $\underline{\text { Crossref }}$

7. Rosenlund B, Kvist U, Ploen L, et al. A comparison between open and percutaneous needle biopsies in men with azoospermia. Hum Reprod. 1998;13:1266-71. Crossref

8. Tournaye H. Surgical sperm recovery for intracytoplasmic sperm injection: which method is to be preferred? Hum Reprod. 1999;14(suppl 1):71-81. Crossref

9. Aridogan IA, Bayazit Y, Yaman, M, ERsoz C, Doran S. Comparison of fine-needle aspiration and open biopsy of testis in sperm retrieval and histopathologic diagnosis. Andrologia. 2003;35:121-5. Crossref

10. Qublan HS, Al-Jader KM, Al-Kaisi NS, Alghoweri AS, Abu-Khait Sa, Abu-Qamar A et al. Fine needle aspiration cytology compared with open biopsy histology for the diagnosis of azoospermia. J Obstet Gynaecol. 2002;22:527-31. Crossref

11. Mercan R, Urman B, Alatas C, Aksoy S, Nuhoglu A, Isiklar A et al. Outcome of testicular sperm retrieval procedures in non-obstructive azoospermia: percutaneous aspiration versus open biopsy. Hum Reprod. 2000;15:1548-51. Crossref

12. Khadra AA, Abdulhadi I, Ghunain S, Kilani Z. Efficiency of percutaneous testicular sperm aspiration as a mode of sperm collection for intracytoplasmic sperm injection in nonobstructive azoospermia. J Urol. 2003;169:603-5. Crossref

13. Harrington TG, Schauer D, Gilber B. Percutaneous testis biopsy: an alternative to open testicular biopsy in the evaluation of the subfertile man. J Urol 1996;156:1647-51. Crossref

14. Schlegel PN, Su LM. Physiological consequences of testicular sperm extraction. Hum Reprod. 1997;12:1688-1692. Crossref

15. Jarrow JP. Clinical significance of intratesticular anatomy. J Urol 1991;145:777-779. Crossref 